\title{
Transactivation of Vimentin by $\beta$-Catenin in Human Breast Cancer Cells
}

\author{
Christine Gilles, ${ }^{1}$ Myriam Polette, ${ }^{1}$ Mélanie Mestdagt, Béatrice Nawrocki-Raby, Philippe Ruggeri, Philippe
} Birembaut, and Jean-Michel Foidart.

Laboratory of Tumor and Developmental Biology, University of Liège, CHU Sart-Tilman B23, B-4000 Liège, Belgium [C. G., M. M, P. R., JM. F.], and Unité LNSERM U.514, Laboratoire Pol Bouin IFR 53, CHU Maison Blanche, Reims, France [M. P., B. N-R, P. B.].

\begin{abstract}
The cytoplasmic and nuclear redistribution of $\beta$-catenin and the de novo expression of vimentin are frequently involved in the epithelial-to-mesenchymal transition associated with increased invasive/migratory properties of epithelial cells. Because $\beta$-catenin can act as a coactivator of transcription through its binding to the T-cell factor (TCF)/lymphoid enhancer factor 1 transcription factor family, we have explored the possibility that $\beta$ catenin/TCF could directly transactivate vimentin. We first compared vimentin expression in relation with the localization of $\beta$-catenin in eight breast cancer cell Unes displaying various degrees of invasiveness and in a model of cell migration using human mammary MCF10A cells. We could thus show a cytoplasmic and/or nuclear distribution of $\beta$-catenin in invasive/migratory cells expressing vimentin, but not in noninvasive/stationary vimentin-negative cell lines. In addition, the human vimentin promoter was found to be up-regulated by $\beta$-catenin and TCF-4 cotransfection. Varying with the cellular background, a diminution of this up-regulation was observed when the putative $\beta$-catenin/TCF binding site of the vimentin promoter was mutated. Our results therefore demonstrate that the vimentin promoter is a target of the $\beta$-catenin/TCF pathway and strongly suggest an implication of this regulation in epithelial cell migration/invasion.
\end{abstract}

Abbreviations: EMT, epithelial-to-mesenchymal transition; TCF, T-cell factor; LEF, lymphoid enhancer factor; MMP, matrix metalloproteinase; EGFP, enhanced green fluorescent protein; DAPI, 4',6-diamidino-2phenylindole; RT-PCR, reverse tran-scription-PCR; GFP, green fluorescent protein.

\section{INTRODUCTION}

During their metastatic conversion, epithelial cells acquire the ability to invade the surrounding tissue and disseminate in secondary organs. Growing evidence emphasizes the concept that the acquisition of migratory/invasive properties by epithelial cells is associated with the gain of mesenchymal characteristics and the loss of epithelial features, a phenomenon referred to as EMT (1-3).

Among the mechanisms largely associated with the metastatic conversion of epithelial cells and the EMT is the loss of E-cadherin-mediated cell adhesion (4). E-cadherin is a transmembrane glycoprotein that mediates homotypic cell-cell contacts between epithelial cells and thereby largely contributes to the cohesive architecture of normal epithelia. The cytoplasmic part of E-cadherin is linked to the actin cytoskeleton via its interaction with its cytoplasmic binding partners, the catenins $(\alpha-, \beta$-, and $\gamma$-catenin). In normal epithelial cells, $\beta$-catenin is mainly localized in E-cadherin complexes and contributes to cell-cell adhesion $(5,6)$. Under particular conditions, $\beta$-catenin accumulates sufficiently in the cytoplasm and can then translocate to the nucleus, where it acts as a transcriptional coactivator through its binding with the members of the TCF/LEF-1 transcription factor family (7). The accumulation of free cytoplasmic $\beta$-catenin and/or its translocation to the nucleus has been associated with epithelial cell migration and the EMT in various physiological or pathological processes including tumor progression $(4-6,8)$. Several genes have now been identified as the target of $\beta$-catenin/TCF transcriptional regulation. These include $M M P-7$ (9-11), uPAR (12), CD44 (13), c-myc (14), cyclin D1 (15), TCF-1 (16), fibronectin (17), slug (18), and the $\gamma 2$ chain of laminin-5 (19). Increasing efforts are being made to identify other targets of $\beta$-catenin transactivation that would facilitate tumor invasion.

Besides the disorganization of E-cadherin- $\beta$-catenin complexes, the de novo expression of vimentin is a mechanism also frequently associated with an EMT and the metastatic conversion of epithelial cells. Vimentin is a type III intermediate filament normally expressed in cells of mesenchymal origin (20). However, numerous data have now demonstrated that vimentin can also be expressed in epithelial cells involved in physiological or

\footnotetext{
${ }^{1}$ C. G. and M. P. contributed equally to this work
} 
pathological processes requiring epithelial cell migration. Vimentin has indeed been described in migratory epithelial cells involved in embryological and organogenesis processes, in placentation, in wound healing, or in tumor invasion $(1,21-24)$. Also, vimentin antisense transfection in vimentin-express-ing breast cell lines was shown to reduce their in vitro invasiveness or migration, strongly emphasizing a functional contribution of vimentin to epithelial cell invasion/migration $(24,25)$. In the same way, an impaired wound healing has been observed in vimentin knockout mice $(26,27)$. Furthermore, a direct or indirect interaction of vimentin with microfilaments and microtubules and more particularly with molecules such as plectin or integrins has been described (28-33). A role of vimentin in the mechanical transduction of signals from the cell surface to the nucleus and in the overall reorganization of the cytoskeleton associated with cell motility and migration has therefore been suggested $(24,26,34,35)$.

A correlation between vimentin expression and a perturbation of E-cadherin-mediated cell adhesion has previously been emphasized. For instance, several studies comparing various breast or lung cell lines have indeed reported that the loss of E-cadherin in highly invasive cell lines is associated with vimentin expression $(36,37)$. Similarly, the induction of an EMT in Madin-Darby canine kidney cells has also been characterized by a decrease of E-cadherin expression and an increase of vimentin expression (38). Vimentin expression and a perturbation of E-cadherin-mediated cell adhesion therefore both appear as hallmarks of EMT-associated events implicated in cell invasion and migration.

In the present study, we examined the possibility that vimentin could be a direct target of the $\beta$-catenin/TCF transactivation pathway in human mammary tumor cells. We thus document an overlap between vimentin expression and nuclear redistribution of $\beta$-catenin in several human mammary tumor cell lines. We also demonstrate the direct transactivation of the vimentin promoter by $\beta$-catenin and TCF- 4 and show the implication of a putative $\beta$-catenin/TCF binding site located $468 \mathrm{bp}$ upstream of the transcription initiation site in this regulation.

\section{MATERIALS AND METHODS}

Cell Culture. All human mammary epithelial cells were obtained from the American Type Culture Collection (Manassas, VA). MCF10A-VP-EGFP cells were generated previously (24) by stable transfection of the VPEGFP plasmid, in which the human vimentin promoter controls the expression of EGFP. BT474, BT20, MCF-7, T47D, MDA-MB-231, MDA-MB-435, BT549, and Hs578T cells were cultured in DMEM containing $10 \%$ FCS. The growth medium of MCF10A cells was composed of Ham's F-12 medium and DMEM [1:3 (v/v)] supplemented with $20 \mu \mathrm{g} / \mathrm{ml}$ adenine, $5 \mu \mathrm{g} / \mathrm{ml}$ insulin, $0.5 \mu \mathrm{g} / \mathrm{ml}$ hydrocortisone, $2 \mathrm{ng} / \mathrm{ml} \mathrm{EGF}, 5 \mu \mathrm{g} / \mathrm{ml}$ transferrin, $1.5 \mathrm{ng} / \mathrm{ml}$ triiodothyronin, and $10 \%$ FCS.

In Vitro Migration Assay. MCF10A cells $\left(5 \times 10^{4}\right)$ were seeded in growth medium inside a 6-mm glass ring placed in the middle of a glass coverslip (22 $\mathrm{mm}$ in diameter). Twenty-four $\mathrm{h}$ after plating, the glass ring was removed, and the cells were covered with growth medium. In this model, the cells migrate as an outgrowth from the confluent area initially delimited by the ring (24).

Immunofluorescence. Cells cultured in the migration assay or as confluent monolayers on glass coverslips were fixed with methanol for $10 \mathrm{~min}$ at $-20^{\circ} \mathrm{C}$. For confocal microscopy analyses of nuclear $\beta$-catenin, cells were fixed in 2.5\% paraformaldehyde in PBS for 30 min and then permeabilized with $0.2 \%$ Triton X-100 for 15 min at room temperature. The coverslips were then saturated for 30 min with $3 \%$ bovine serum albumin in PBS. After intermediate washes in PBS, monolayers were incubated for $1 \mathrm{~h}$ with a monoclonal antibody to vimentin (clone V9; Dako, Glostrup, Denmark), E-cadherin (BD Transduction Laboratories, San Jose, CA), or $\beta$-catenin (BD Transduction Laboratories). Cells were then exposed to a tetramethylrhodamine isothiocyanate-conjugated rabbit antimouse antibody (Dako). After incubation with the different antibodies, nuclei were labeled with DAPI $(1 \mu \mathrm{g} / \mathrm{ml})$ for $20 \mathrm{~min}$. The coverslips were then mounted with Aquapolymount antifading solution (Agar) onto glass slides and observed under a Zeiss fluorescence microscope or with a MRC 600 confocal laser scanning microscope (Bio-Rad, Richmond, CA).

Plasmids. The vimentin promoter luciferase reporter vector (VimPro) was constructed by cloning an EcoRIBam HI 1.5-kb fragment of the human vimentin 5' upstream region into the firefly luciferase reporter plasmid pGL-3 (Promega, Madison, WI). This fragment, described and cloned as the human vimentin promoter (39), was isolated from pG4F1EM1.5, kindly provided by Dr. S. Rittling (Temple University Medical School, Philadelphia, PA). The GenBank accession number for the human vimentin promoter is M17888. This human vimentin promoter contains a consensus $\beta$-catenin/TCF binding site (5'-A/T-A/T-CAAAG-3') in an inverted position located $468 \mathrm{bp}$ upstream of the transcription initiation start (5'-CTTTGTT-3'). We also constructed a 
reporter vector containing a mutated $\beta$-catenin/TCF binding site (5'-CTTT-GGC-3'; VimPro Mut) using the QuikChange site-directed mutagenesis kit (Stratagene, La Jolla, CA) and the following primers, 5'CAATCTCAG-GCGCTCTTTGGCTCTTTCTCCGCGACTTCAG-3' and 5'-CTGAAGTCGCGGAGAAAGAGCCAAAGAGCGCCTGAGATTG-3'. Such a 2-nucleotide alteration in the $\beta$-catenin/TCF binding site mutation has previously been described to dramatically reduce $\beta$-catenin/TCF signaling $(40,41)$.

The expression vector for $\beta$-catenin expressing a mutated form of $\beta$-catenin that is less susceptible to degradation was kindly provided by Drs. K. Orford and S. Byers (Lombardi Cancer Center, Georgetown University, Washington, DC; Ref 42). The expression vector encoding the human TCF-4 (pCDNA-hTCF4) and the TOPFLASH and FOP-FLASH plasmids containing three wild-type (5'-CCTTTGATC-3'; TOP-FLASH) or mutated (5'-CCTTTGGCC-3'; FOP-FLASH) copies of the $\beta$-catenin/TCF binding sites upstream of a minimal c-fos promoter driving the firefly luciferase expression were a kind gift from Dr. H. C. Clevers [University Hospital, Utrecht, the Netherlands $(40,41)]$.

Transient Transfections and Reporter Assay. Transient transfections were performed with FuGENE transfection reagent (Roche, Branchburg, NJ) on 50,000 cells plated in a 24-well plate half an hour before the addition of the DNA/FuGENE mixture.

For the determination of the TOP-FLASH/FOP-FLASH activity, each well was supplemented with a mixture containing $20 \mu \mathrm{l}$ of serum-free DMEM, $0.6 \mu \mathrm{l}$ of FuGENE, and $0.5 \mu \mathrm{g}$ of the TOP-FLASH or FOP-FLASH firefly luciferase reporter plasmid and $0.8 \mathrm{ng}$ of the Renilla luciferase reporter phRG-TK (Promega) as an internal control.

For $\beta$-catenin/TCF-4 induction, each well was incubated with a mixture containing $20 \mu 1$ of serum-free DMEM, $0.6 \mu \mathrm{l}$ of FuGENE, $0.15 \mu \mathrm{g}$ of the firefly luciferase reporter plasmid (either the VimPro, VimProMut, TOPFLASH, or FOP-FLASH reporter construct), $0.15 \mu \mathrm{g}$ of the $\beta$-catenin expression vector (or the corresponding empty vector), $0.15 \mu \mathrm{g}$ of the TCF-4 expression vector (or the corresponding empty vector), and $0.8 \mathrm{ng}$ of the Renilla luciferase vector phRG-TK.

Twenty-four h after transfection, the cells were lysed in $50 \mu \mathrm{l}$ of passive lysis buffer, and the luciferase activity was determined with a luminometer using the Dual Luciferase Assay System (Promega) on $20 \mu 1$ of lysate. For each experiment, the firefly luciferase activity was normalized to the activity of the Renilla luciferase used as internal control. The activities of the TOP-FLASH and the FOP-FLASH reporter constructs were expressed as normalized relative light units. For $\beta$-catenin/TCF-4 induction experiments, results were expressed as fold induction. Fold induction was determined by normalizing each firefly luciferase value to the Renilla luciferase internal control value and by dividing these normalized values with the mean normalized value of the corresponding reporter construct transfected with the empty expression vectors. Each experiment was performed at least three times in triplicate. Data are expressed as means $\pm \mathrm{SD}$. A one-way ANOVA was performed, and $P<$ 0.05 was considered significant.

Transient Transfections and Vimentin Expression Analyses. To study the regulation of the endogenous vimentin gene by $\beta$-catenin/TCF-4 complexes, transient transfections of $\beta$-catenin and TCF- 4 were performed with Lipofectamine 2000 transfection reagent (Invitrogen, Carlsbad, CA) on 200,000 MCF10A cells plated in 6well plate. Twenty-four $\mathrm{h}$ after plating, transfection was carried out as recommended by the manufacturer by adding to each well a mixture containing $500 \mu \mathrm{l}$ of serum-free medium, $3 \mu \mathrm{l}$ of Lipofectamine $2000,1 \mu \mathrm{g}$ of the $\beta$-catenin expression vector, and $1 \mu \mathrm{g}$ of the TCF-4 expression vector. Controls were generated by transfecting the cells with the corresponding empty vectors. Twenty-four $\mathrm{h}$ after transfection, total RNA extraction was performed with the RNA isolation kit (Roche). RT-PCR was performed using 10 ng of total RNA, the Gene Amp Thermostable RNA PCR Kit (Perkin-Elmer, Foster City, CA), and pairs of primers for human vimentin and for 28S rRNA control amplification (Eurogentec, Seraing, Belgium). Forward and reverse primers for human vimentin and 28S were designed as follows: (a) human vimentin primers, 5'-GACAATGCGTCTCTGGCACGTCTT-3' (forward) and 5'-TCCTCCGCCTCCTGCAGGTTCTT-3' (reverse); (b) 28S primers, 5'GTTCACCCACTAATAGGGAACGTGA-3' (forward) and 5'-GGATTCTGACTTAGAGGCGTTCAGT-3' (reverse). Reverse transcription was performed at $70^{\circ} \mathrm{C}$ for $15 \mathrm{~min}$. Amplification cycles were as follows: $15 \mathrm{~s}$ at $94^{\circ} \mathrm{C} ; 20 \mathrm{~s}$ at $68^{\circ} \mathrm{C}$; and $10 \mathrm{~s}$ at $72^{\circ} \mathrm{C}$ Twenty-nine cycles were allowed for vimentin amplification, and 15 cycles were allowed for $28 \mathrm{~S}$ amplification. Products were separated on acrylamide gels, stained with SYBR Gold (Molecular Probes, Eugene, OR), and quantified by fluori-metric scanning (LAS-1000; Fuji, Stamford, CT). The values obtained for vimentin amplification were normalized with values obtained for $28 \mathrm{~S}$ amplification. Each experiment was performed three times. Data are expressed as means $\pm \mathrm{SD}$. 


\section{RESULTS}

Vimentin Expression Associates with the Cytoplasmic and Nuclear Distribution of $\beta$-Catenin. To test the possibility that the $\beta$-catenin transactivation pathway could regulate vimentin expression, we first examined these two proteins by immunofluorescence in a set of eight human breast tumor cell lines (BT474, BT20, T47D, MCF7, MDA-MB-231, BT549, Hs578T, and MDA-MB-435). These cell lines were chosen because they previously allowed establishment of a clear-cut correlation between the loss of E-cadherin expression, the expression of vimentin, and high invasive abilities $(36,43-45)$. Indeed, as reported in these studies and as confirmed in our present study (data not shown), BT474, BT20, MCF-7, and T47D cells are epithelioid in shape, noninvasive in Boyden chamber assay, and E-cadherin positive and vimentin negative, whereas MDA-MB-231, BT549, Hs578T, and MDA-MB-435 cells are rather fibroblastoid in shape, vimentin positive, E-cadherin negative, and highly invasive. Such a differential expression of vimentin is illustrated for MCF-7 epithelioid cells and Hs578T fibroblastoid cells in Fig. 1, $A$ and $B$. $\beta$-Catenin immunofluorescence analyses and confocal microscopy observations on these characterized cell lines clearly revealed that $\beta$-catenin is mainly found as an intense membrane staining in E-cadherin-positive, vimentin-negative, noninvasive cells, as illustrated for MCF-7 cells in Fig. $1 C$. In contrast, $\beta$-catenin staining was mainly detected in the cytoplasm of vimentin-positive, invasive cell lines, as illustrated for $\mathrm{Hs} 578 \mathrm{~T}$ in Fig. $1 C$. Examining various optical sections, we could also observe that vimentin-expressing Hs578T cells displayed a nuclear labeling for $\beta$-catenin coinciding with DAPI staining (Fig. 1C). In contrast, vimentin-negative MCF-7 cells clearly never showed any nuclear $\beta$-catenin.

Next we studied E-cadherin and $\beta$-catenin in relation with vimentin expression in a migration assay that we previously used to demonstrate the transient expression of vimentin during epithelial cell migration (24). Briefly, in this migration assay, MCF10A cells are plated at a high density in a glass ring and migrate as an outgrowth after the removal of the ring. In contrast to BT474, BT20, MCF-7, or the T47D cells, which constitutively lack vimentin expression, MCF10A cells were previously shown to be inducible for vimentin expression in this assay. Indeed, using videomicroscopy, we demonstrated previously that cells at the periphery of the outgrowth specifically express vimentin and are involved in an orientated migration. Cells distant from that periphery are basically stationary and do not express vimentin (24). In the present study, we used MCF10A stably transfected with a plasmid containing the vimentin promoter controlling the expression of the GFP gene (VP-EGFP MCF10A cells). The use of this assay allowed us to establish a clearer correlation between the disorganization of E-cadherin- $\beta$-catenin complexes and the activation of the vimentin promoter. We could indeed show a diminution of E-cadherin and $\beta$-catenin membrane-associated honeycomb stainings in migratory cells that expressed vimentin at the periphery of the outgrowth (Fig. $2 A$ ). GFP expression was preferentially seen in cells that did not display a typical honeycomb $\beta$-catenin staining, demonstrating the specific activation of the vimentin promoter in these cells (Fig. 2A). Fig. $2 A$ also shows that the expression of the vimentin protein detected by immunofluorescence coincides well with the activation of the vimentin promoter. Furthermore, confocal analyses showed that migratory cells at the periphery of the outgrowth display an intense cytoplasmic and nuclear $\beta$-catenin staining (coinciding with DAPI labeling). In contrast, cells distant from that periphery mostly displayed a honeycomb $\beta$-catenin distribution and no nuclear staining (Fig. $2 B$ ). These data, taken together, clearly correlate a cytoplasmic and nuclear localization of $\beta$-catenin in invasive or migratory epithelial cells with vimentin expression.

Vimentin-positive Cells Display an Elevated $\beta$-Catenin/TCF Transcriptional Activity. We then compared the ability of vimentin-expressing cells versus vimentin-negative cells to transactivate a luciferase reporter plasmid containing wild-type (TOP-FLASH) or mutated (FOP-FLASH) $\beta$-catenin/TCF binding sites as regulatory elements. For this purpose, we examined vimentin-negative BT474, BT20, and MCF-7 cells and compared them with BT549 and Hs578T cells, which display a constitutive expression of vimentin, and MCF$10 \mathrm{~A}$ cells, which are also able to express vimentin. We observed that the three cell lines able to express vimentin (BT549, Hs578T, and MCF10A cells) displayed a higher ability to transactivate the TOP-FLASH reporter plasmid than the vimentin-negative BT474, BT20, and MCF-7 cells (Fig. 3). In all cell lines displaying a high TOP-FLASH activity, an important decreased activity was reported for the FOP-FLASH plasmid. In accordance with our findings showing a preferential cytoplasmic and nuclear localization of $\beta$-catenin in vimentinexpressing cells, these data clearly suggested that vimentin-expressing cells have a stronger $\beta$-catenin/TCF transcriptional activity than the vimentin-negative ones. 
Fig. 1: Preferential cytoplasmic and nuclear localization of $\beta$-catenin in vimentin-expressing cell lines. A, phasecontrast microscopy images of MCF-7 and Hs578T monolayers. Bar, $76 \mu \mathrm{m}$. B, vimentin immunostainings on MCF-7 and Hs $578 T$ monolayers. Bar, $42 \mu \mathrm{m}$. C, confocal microscopy analyses of $\beta$-catenin labeling on MCF-7 and Hs578T monolayers. Serial optical sections were taken from the basal to the apical surface of the cells (section 1-75) and clearly revealed a cytoplasmic $\beta$-catenin labeling (in a basal section) and a nuclear labeling (in an intermediate section) on Hs578T cells, whereas $\beta$-catenin was found mostly as an intense labeling at the

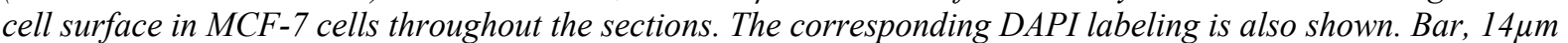

MCF-7

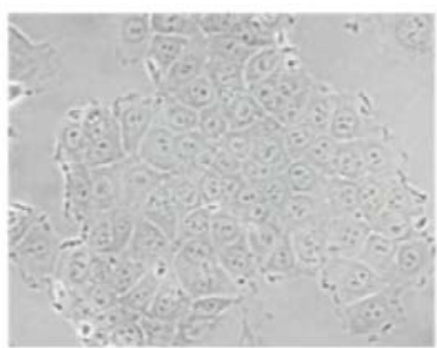

A

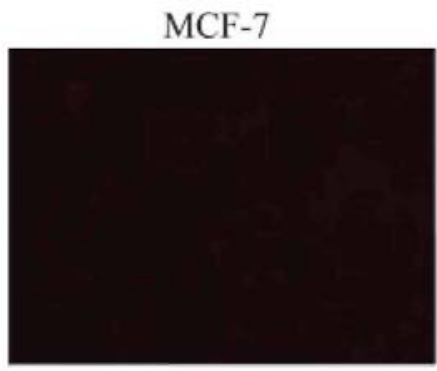

B
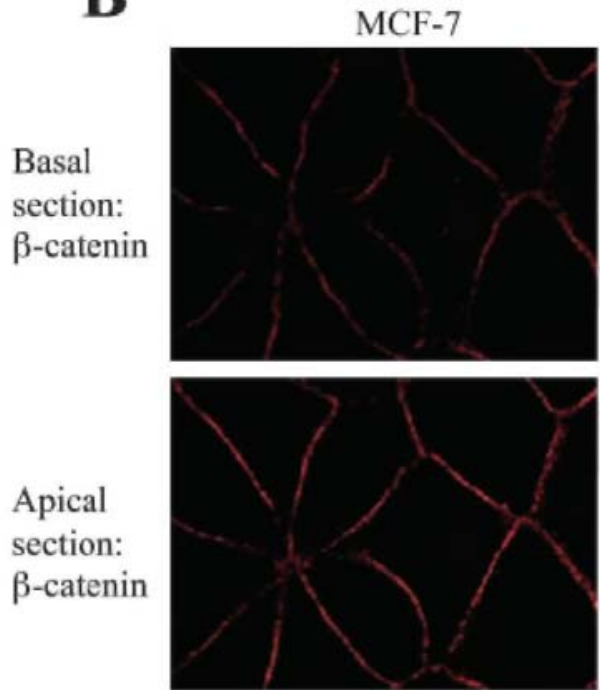

Apical section: $\beta$-catenin

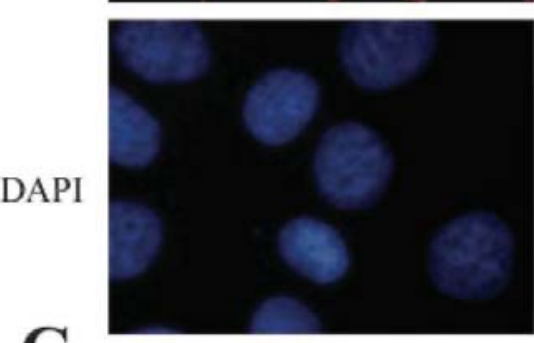

Hs578T

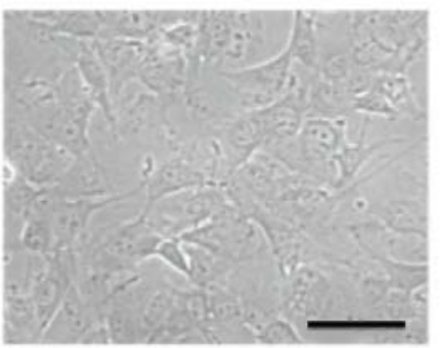

Hs578T

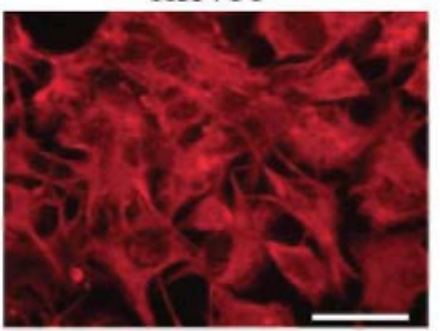

Hs578T
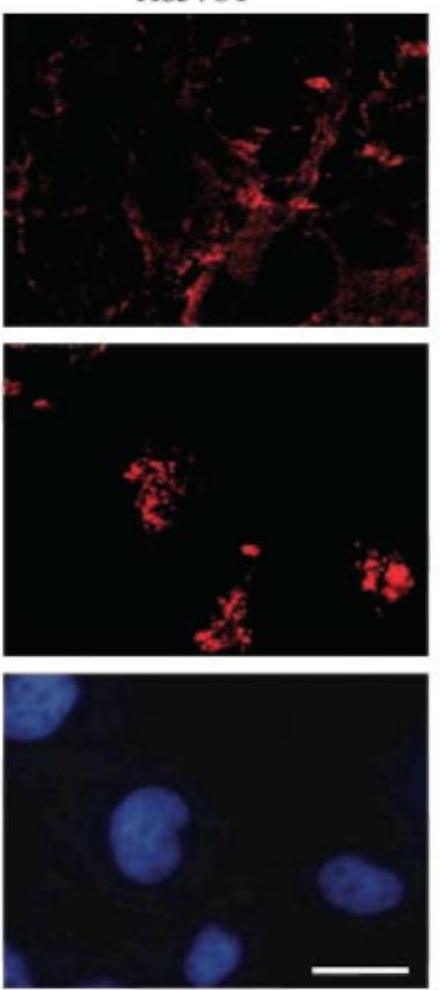
Fig. 2: The activation of the vimentin promoter coincides with a cytoplasmic and a nuclear localization of $\beta$ catenin in migratory MCF10A cells. A, single or double visualization (Merge) of vimentin. $\beta$-catenin, or Ecadherin immunolabeling (in red) and GFP expression (in green) in VP-EGFP MCF10A cells plated in the migration assay. Bar, $84 \mu \mathrm{m}$. B, confocal microscopy analyses of $\beta$-catenin labeling on MCF10A cells plated in the migration assay. Serial optical sections were taken from the basal to the apical surface of the cells (section $1-75$ ) and clearly revealed a cytoplasmic $\beta$-catenin labeling (in a basal section) and a nuclear labeling (in an intermediate section) in migratory cells at the periphery of the outgrowth, whereas $\beta$-catenin was found mostly as an intense labeling at the cell surface throughout the sections in stationary cells distant from that periphery. The corresponding DAPI labeling is also shown. A white line delineates the edge of the outgrowth. Bar, $24 \mu \mathrm{m}$

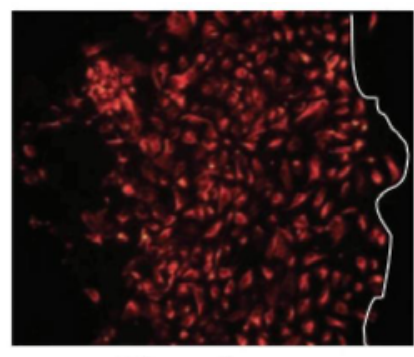

Vimentin

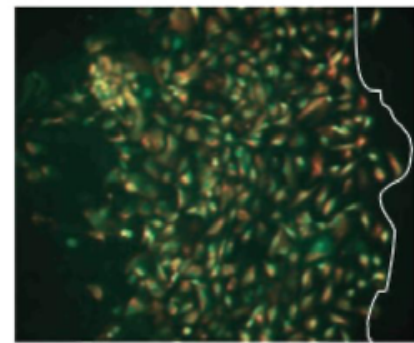

Merge Vim/GFP

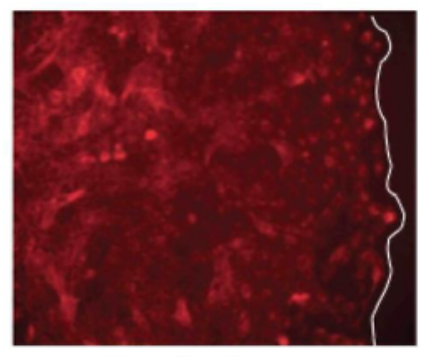

E-cadherin

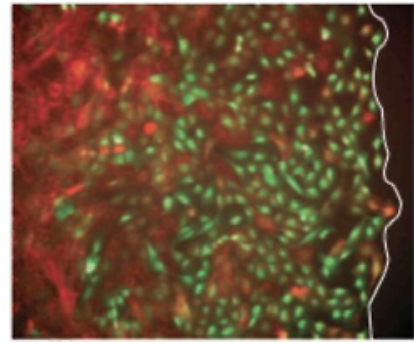

Merge E-cad/GFP

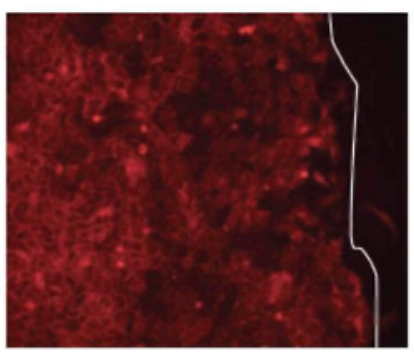

$\beta$-catenin

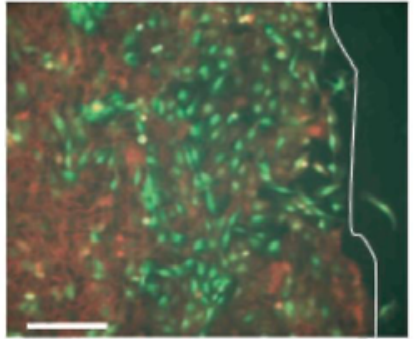

Merge $\beta$-cat/GFP

A
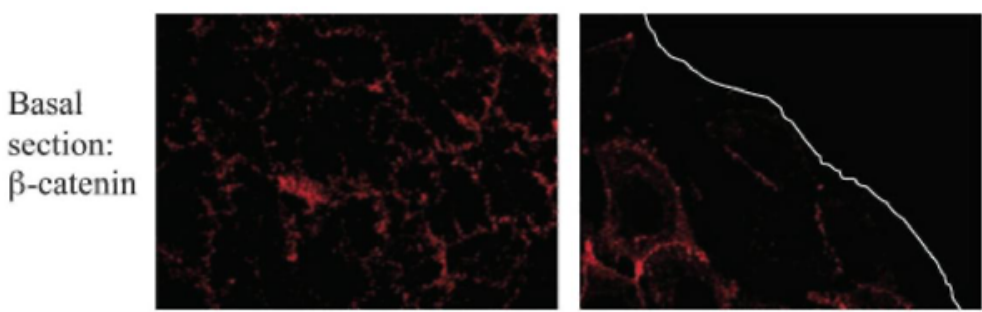

Apical

section:

$\beta$-catenin
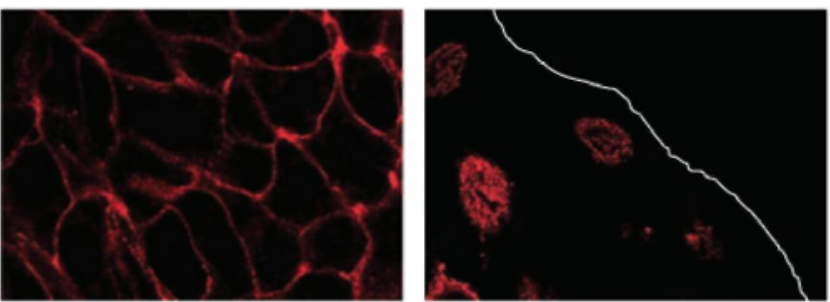

DAPI
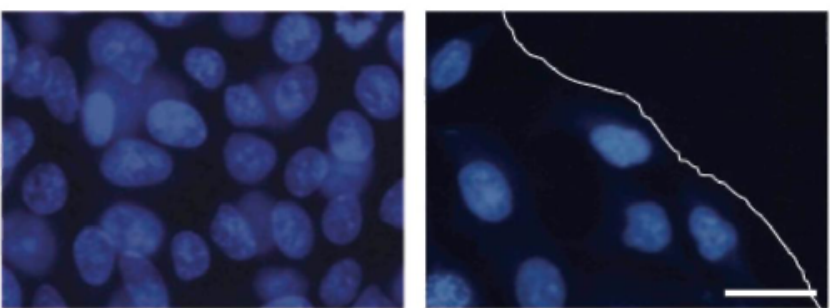

Distant from the outgrowth periphery

Outgrowth periphery 
Fig. 3: Vimentin-expressing cell lines display a higher $\beta$-catenin/TCF activity than vimentm-negative cell lines. Different cell lines (BT474, BT20, MCF-7, BT549, Hs578T: and MCF10A cells) were transfected with the TOPFLASH or FOP-FLASH firefly luciferase reporter plasmids. Data are expressed as relative light units (RLUS) normalized to the cotransfected Renilla luciferase-encoding phRG-TK plasmid

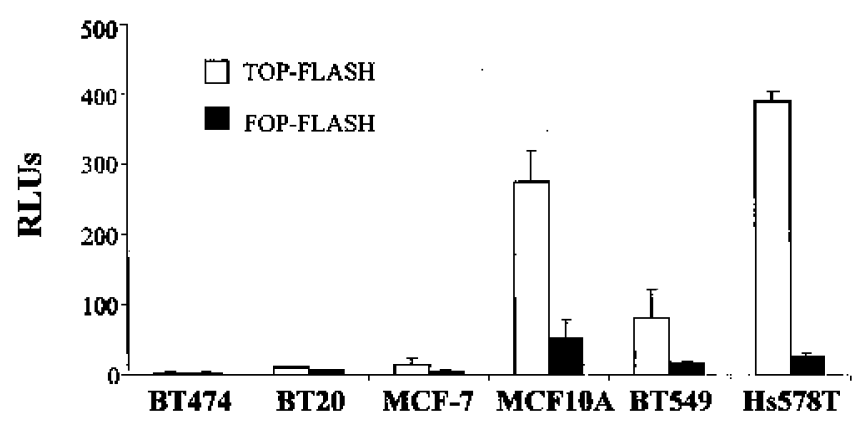

Fig. 4: $\beta$-Catenin and TCF-4 transactivate the human vimentin promoter. A, the VimPro or the VimProMut reporter plasmid was cotransfected with the empty expression vectors (Lane 1$)$, the $\beta$-catenin expression vector (Lane 2), the TCF-4 expression vector (Lane 3), or the $\beta$-catenin and the TCF-4 expression vectors (Lane 4). Data are presented as fold induction relative to the cotransfection of the reporter plasmid (either VimPro or VimProMut) with the empty expression vectors (Lane 1). B, representative RT-PCR analysis of vimentin $m R N A$ (Vim) and 28S in MCF10A cells cotransfected with $\beta$-catenin and TCF-4 ( $\beta$-cat/TCF-4) or with the corresponding empty expression vectors (Cont) $C$, quantification of RT-PCR analyses of vimentin normalized with the $28 S$ values in three independent transfection experiments as described in B. Data are expressed as fold induction relative to the control transfection (Cont).
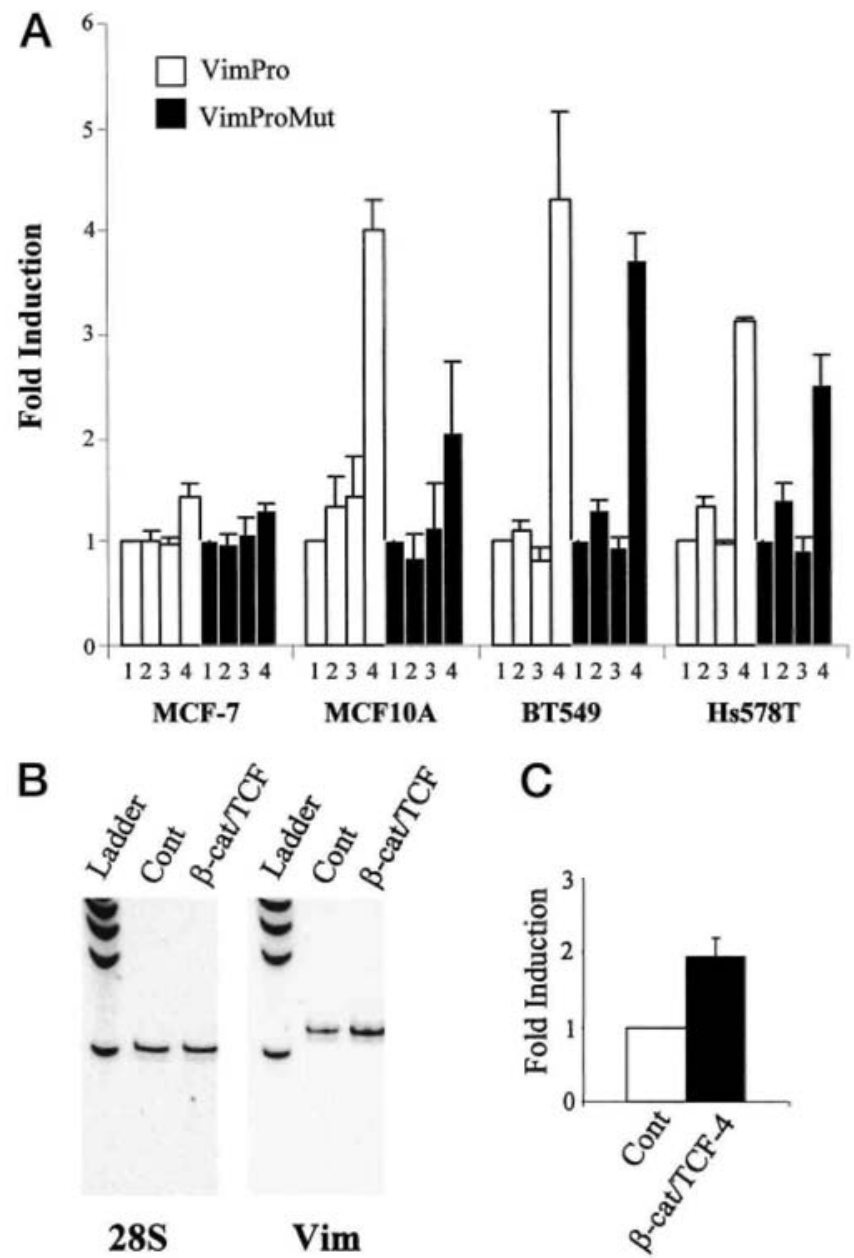
$\boldsymbol{\beta}$-Catenin/TCF Regulates the Vimentin Promoter. To examine the possibility that $\beta$-catenin could directly regulate the transcription of vimentin, we used a luciferase reporter plasmid containing the human vimentin promoter (VimPro-Luc) in transient transfection experiments in the vimentin-negative MCF-7 cell line and in three vimentin-expressing cell lines (MCF10A, BT549, and Hs578T). The human vimentin promoter contains a putative $\beta$-catenin/TCF binding site 468 bp upstream of the transcription start site. We also generated a reporter plasmid containing the vimentin promoter mutated at the putative $\beta$-catenin/TCF binding site (VimProMut-Luc). We thus performed cotransfection experiments using the VimPro-Luc and the VimProMut-Luc with expression vectors encoding $\beta$-catenin and/or a member of the LEF-1/TCF transcription factor family, TCF-4.

Cotransfections of $\beta$-catenin and TCF-4 led to a clear activation (between 3.1- and 4.3-fold induction) of the wild-type vimentin promoter in BT549, Hs578T, and MCF10A cells (Fig. 44). The transfection of $\beta$-catenin or TCF-4 alone did not significantly activate the wild-type vimentin promoter. Interestingly, only a slight activation of the vimentin promoter was observed in vimentin-negative MCF-7 cells. The use of the mutated promoter revealed a reduction of the activation obtained with $\beta$-catenin and TCF-4. This reduction was particularly obvious in MCF10A cells, was weak in Hs578T cells, and was not significant in BT549 cells (Fig. 44). In agreement with our data obtained with the promoter reporter constructs, we also found that co-transfection of $\beta$ catenin and TCF-4 generated a 1.95-fold increase of the vimentin mRNA level in MCF10A cells (Fig. 4, B and C).

Because the induction of the vimentin promoter by $\beta$-catenin/ TCF-4 was clearly dependent on the cellular background (no clear induction in MCF-7 cells), we further examined the influence of this background on the $\beta$ catenin/TCF-4 activation of the TOP-FLASH and FOP-FLASH reporter plasmid. We observed an increase of the overall $\beta$-catenin/TCF activity in all of the cell lines by the $\beta$-catenin/ TCF- 4 cotransfection that was diminished with the FOP-FLASH reporter plasmid. Interestingly, this induction was the highest in MCF-7 cells (Fig. 5).

Fig. 5: $\beta$-Catenin and TCF-4 activate the TOP-FLASH reporter plasmid. The TOP-FLASH or FOP-FLASH reporter plasmid was cotransfected with the empty expression vectors (Lane 1) or with the $\beta$-catenin and TCF-4 expression vectors (Lane 2). Data are presented as fold induction relative to the cotransfection of the reporter plasmid (either TOP-FLASH or FOP-FLASH) with the empty expression vectors

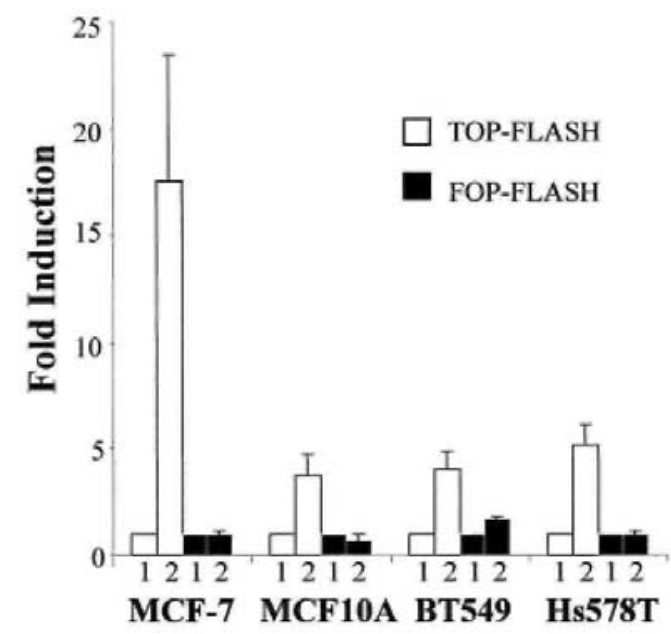

\section{DISCUSSION}

In the present study, we demonstrate that vimentin is a target of the $\beta$-catenin/TCF pathway and emphasize the implication of such a regulation during epithelial cell migration. We indeed showed that $(a)$ a cytoplasmic and nuclear localization of $\beta$-catenin in mammary human epithelial cell lines is associated with vimentin expression and a high invasive/migratory potential, (b) vimentin-positive invasive cell lines display a high $\beta$-catenin/TCF transactivating ability, and (c) the vimentin promoter contains a putative $\beta$-catenin/TCF binding site and is a direct target of $\beta$-catenin/TCF transcriptional regulation. 
Examining several human breast cancer cell lines, we indeed showed that the invasive cell lines expressing vimentin displayed a cytoplasmic and nuclear $\beta$-catenin labeling. In contrast, the vimentin-negative, noninvasive cell lines showed membrane-associated $\beta$-catenin. Such an overlap between vimentin expression and $\beta$-catenin redistribution from the cell membrane was even better emphasized in the migration assay with the MCF10A cells. Indeed, this dynamic model clearly allowed us to discriminate a subpopulation of migratory, vimentinpositive cells within the same cell line. We were thus able to demonstrate that a diminution of the membranelocalized E-cadherin/ $\beta$-catenin and a cytoplasmic and nuclear distribution of $\beta$-catenin specifically in the migratory subpopulation of cells at the periphery of the outgrowth coincide with the transactivation of the vimentin promoter and vimentin expression. In support of our findings, a cytoplasmic accumulation and/or a nuclear translocation of $\beta$-catenin has been associated with an EMT and enhanced migration or invasion in several in vitro systems. For instance, a cytoplasmic accumulation and/or a nuclear translocation of $\beta$-catenin has been described during the EMT and/or migration induced by various means in NBTП or Madin-Darby canine kidney cells (46-49), two cell lines in which an EMT has been associated with vimentin expression in independent studies. Taken together with these data, our findings therefore clearly emphasize an overlap between a cytoplasmic and/or nuclear redistribution of $\beta$-catenin and vimentin expression in invasive and migratory epithelial cells.

We have also shown that the vimentin-expressing cells displayed a higher $\beta$-catenin/TCF activity than the vimentin-negative cells using the TOP-FLASH reporter plasmid. Similarly, Murakami et al. (50), comparing highly and poorly migratory melanoma cell lines, also showed a nuclear accumulation and an enhanced $\beta$ catenin/TCF activity in the highly migratory cell lines. It should be noted that van de Wetering et al. (51) reported a weak $\beta$-catenin/TCF activity in some of the breast cell lines we used when compared with colon cancer SW480 cells. Nevertheless, our results showing a difference of $\beta$-catenin/TCF activity in the invasive versus the noninvasive cell lines and a reduction of this activity using the FOP-FLASH reporter plasmid containing mutated $\beta$-catenin/TCF binding sites strongly support a role of the $\beta$-catenin/TCF pathway in the invasive vimentin-expressing breast cell lines.

In addition to the overlap between $\beta$-catenin redistribution, enhanced $\beta$-catenin/TCF activity, and vimentin expression, the human vimentin promoter was found to be directly activated by $\beta$-catenin/ TCF- 4 transfections. We indeed reported an up to 4 -fold induction of the vimentin promoter by cotransfection of $\beta$-catenin and TCF4 , but not by transfection of $\beta$-catenin or TCF-4 alone, in the vimentin-expressing cells. Similarly, a synergistic activation by $\beta$-catenin and TCF- 4 of the human promoters of MMP-7 and of the $\gamma 2$ chain of laminin-5 have been reported, whereas $\beta$-catenin or TCF-4 alone had weaker effects $(9,19)$. Furthermore, a repressive effect of TCF/LEF-1 factors has also been described as shown on the mouse MMP-7 promoter and the human myc promoter, suggesting that $\beta$-catenin could act as a transcriptional coactivator by abrogating TCF-mediated repression $(10,14)$. Other studies have also shown that the induction of the mouse or human MMP-7 promoters by $\beta$-catenin varies in different cellular backgrounds, also emphasizing that $\beta$-catenin over-expression is not always sufficient $(10,11)$. In addition, we also showed that the mutation of the putative $\beta$-catenin/TCF binding site identified in the human vimentin promoter diminished the induction of the promoter by $\beta$-catenin and TCF4 , particularly in MCF10A cells. Nevertheless, the mutation of the putative $\beta$-catenin/TCF binding site in the human vimentin promoter did not completely suppress the $\beta$-catenin/TCF-4-mediated induction of the vimentin promoter. In addition to the contribution of the putative $\beta$-catenin/TCF binding site, the implication of other binding sites similar in sequence cannot be excluded. It is also possible that other $\beta$-catenin/TCF targets could in turn regulate the vimentin promoter. Together with our findings that vimentin mRNA level is increased in MCF10A cells cotransfected with $\beta$-catenin and TCF-4, these data therefore demonstrate that the human vimentin promoter can be a direct target of the $\beta$-catenin/ TCF-4 pathway and suggest a partial contribution of the putative $\beta$-catenin-TCF/LEF binding site in this regulation.

Our results showing the transactivation of the vimentin promoter by $\beta$-catenin/TCF-4 in vimentin-expressing cells but not in MCF-7 cells also emphasize the influence of the cellular context on the $\beta$-catenin/TCF transcriptional regulation. Together with our findings that the TOP-FLASH vector responds to $\beta$-catenin/TCF-4 in MCF-7 cells but not the vimentin promoter, these data emphasize the importance of the level of endogenous transcriptional activators or repressors on the regulation of a given promoter. Also in agreement with this concept are our findings that the diminution of $\beta$-catenin/TCF-4-induced activation with the mutated vimentin promoter varies from one cell line to another. Accordingly, multiple positive but also negative regulatory elements and factors have been shown to influence the regulation of the vimentin promoter (39,

52-60). For instance, a member of the ets transcription factor family (PEA3) and AP-1 have been directly shown to contribute to the differential regulation of the human vimentin promoter in invasive, vimentin-positive cells versus noninvasive, vimentin-negative cells $(57,61,62)$. Also, a synergy between $\beta$-catenin-TCF/LEF 
complexes and other transcription factors (including AP-1 and PEA-3) has been described in the regulation of the human MMP-7 promoter and the promoter of the $\gamma 2$ chain of laminin-5 $(11,19)$. Adding to that complexity, TCF proteins have also been shown to bind the corepressor groucho $(63,64)$. The responsiveness of the human vimentin promoter to the $\beta$-catenin/TCF pathway therefore clearly depends not only on the structure of the promoter itself, but also on the cellular context. This context is defined by the levels of endogenous $\beta$-catenin and TCF/LEF factors but also by the levels of other transcriptional activators or repressors that could directly or indirectly interact with this complex. Nevertheless, if the cellular context obviously modulates the $\beta$-catenin/TCF regulation of the human vimentin promoter, our data showing an induction of vimentin promoter activity by $\beta$ catenin/TCF-4 indicate that the human vimentin promoter can be a direct target of the $\beta$-catenin/TCF pathway.

In conclusion, our results clearly demonstrate the regulation of the vimentin promoter by the $\beta$-catenin/TCF pathway. Our findings that the accumulation of cytoplasmic/nuclear $\beta$-catenin localization and vimentin expression coincide and are restricted to invasive or effectively migrating cells suggest a functional contribution of this regulation in epithelial cell migration.

Because of the functional role of vimentin in cell migration, the regulation of vimentin by the $\beta$-catenin/TCF pathway has implications in all processes requiring epithelial cell migration, including tumor invasion. It is of particular interest to note that an increasing number of $\beta$-catenin target genes [such as $M M P-7(9,11), C D 44$ (13), uPAR (12), slug (18) or the $\gamma 2$ chain of laminin-5 (19)] have been largely involved in the acquisition of migratory/invasive properties by epithelial cells. Together with these data, our present findings that $\beta$-catenin can directly regulate vimentin support a more general role of $\beta$-catenin as a regulator of EMT-associated events occurring during cell migration.

\section{ACKNOWLEDGMENTS}

We thank Dr. S. Rittling for the generous gift of the human vimentin promoter; Dr. H. Clevers for TOP-FLASH, FOP-FLASH, and TCF-4 expression vector; and Drs. K. Orford and S. Byers for the $\beta$-catenin expression vector.

The costs of publication of this article were defrayed in part by the payment of page charges. This article must therefore be hereby marked advertisement in accordance with 18 U.S.C. Section 1734 solely to indicate this fact.

Supported by grants from the Communauté Française de Belgique (Actions de Recherches Concertées), the Commission of European Communities, the Fonds de la Recherche Scientifique Médicale, the Fonds National de la Recherche Scientifique (FNRS, Belgium), the Fédération Belge Contre le Cancer, the CGRI-FNRS- $\Gamma$ NSERM Coopération, the Fonds Spéciaux de la Recherche (University of Liège), the Centre Anticancéreux près l'Université de Liège, the FB Assurances, the Fondation Léon Frédéricq (University of Liège), the DGTRE from the Région Wallonne, the Fonds d'Investissements de la Recherche Scientifique (CHU, Liège, Belgium), the Interuniversity Attraction Poles (IAP) from the Federal Office for Scientific, Technical and Cultural Affairs (OSTC, Brussels, Belgium), Rhône-Poulenc Rorer Pharmaceuticals (Collegville, PA), and Roche Diagnostics GmbH (Penzberg, Germany). C. G. is a Research Associate from the FNRS (Belgium).

\section{REFERENCES}

1. Gilles, C, and Thompson, E. W. The epithelial to mesenchymal transition and metastatic progression in carcinoma. Breast J., 2: 83-96, 1996.

2. Savagner, P. Leaving the neighborhood: molecular mechanisms involved during epithelial-mesenchymal transition. Bioessays, 23: 912 $923,2001$.

3. Thiery, J. P. Epithelial-mesenchymal transitions in tumour progression. Nat. Rev. Cancer, 2: 442-454, 2002.

4. Van Aken, E., De Wever, O., Correia da Rocha, A. S., and Mareel, M. Defective E-cadherin/catenin complexes in human cancer. Virchows Arch., 439: 725-751, 2001

5. Hecht, A., and Kemler, R. Curbing the nuclear activities of $\beta$-catenin. Control over Wnt target gene expression. EMBO Rep., 1: 24-28, 2000 .

6. Gottardi, C. J., and Gumbiner, B. M. Adhesion signaling: how $\beta$-catenin interacts with its partners. Curr. Biol., 11: R792-R794, 2001. 
7. Behrens, J., von Kries, J. P., Kuhl, M., Bruhn, L., Wedlich, D., Grosschedl, R., and Birchmeier, W. Functional interaction of $\beta$-catenin with the transcription factor LEF-1. Nature (Lond.), 382: 638-642, 1996.

8. Conacci-Sorrell, M., Zhurinsky, J., and Ben Ze'ev. A. The cadherin-catenin adhesion system in signaling and cancer. J. Clin. Investig., 109: 987-991, 2002.

9. Brabletz, T., Jung, A., Dag, S., Hlubek, F., and Kirchner, T. $\beta$-Catenin regulates the expression of the matrix metalloproteinase-7 in human colorectal cancer. Am. J. Pathol., 155: 1033-1038, 1999.

10. Crawford, H. C, Fmgleton, B. M., Rudolph-Owen, L. A., Goss, K. J., Rubmfeld, B., Polakis, P., and Matrisian, L. M. The metalloproteinase matrilysin is a target of $\beta$-catenin transactivation in intestinal tumors. Oncogene, 18: 2883-2891, 1999.

11. Crawford, H. C, Fingleton, B., Gustavson, M. D., Kurpios, N, Wagenaar, R. A., Hassell, J. A., and Matrisian, L. M. The PEA3 subfamily of Ets transcription factors synergizes with $\beta$-catenin-LEF-1 to activate matrilysin transcription in intestinal tumors. Mol. Cell. Biol., 21 : $1370-1383,2001$

12. Mann, B., Gelos, M., Siedow, A., Hanski, M. L., Gratchev, A., Ilyas, M., Bodmer, W. F., Moyer, M. P., Riecken, E. O., Buhr, H. J., and Hanski, C. Target genes of $\beta$-catenin-T cell-factor/lymphoid-enhancer-factor signaling in human colorectal carcinomas. Proc. Natl. Acad. Sci. USA, 96: 1603-1608, 1999.

13. Wielenga, V. J., Smits, R., Kormek, V., Smit, L., Kielman, M., Fodde, R., Clevers, H., and Pals, S. T. Expression of CD44 in Ape and Tcf mutant mice implies regulation by the WNT pathway. Am. J. Pathol., 154: 515-523, 1999.

14. He, T. C, Sparks, A. B., Rago, C, Hermekmg, H., Zawel, L., da Costa, L. T., Morm, P. J., Vogelstein, B., and Kinzler, K. W. Identification of c-MYC as a target of the APC pathway. Science (Wash. DC), 281: 1509-1512, 1998.

15. Tetsu, O., and McCormick, F. $\beta$-Catenin regulates expression of cyclin Dl in colon carcinoma cells. Nature (Lond.), 398: 422-426, 1999.

16. Roose, J., Huls, G., van Beest, M., Moerer, P., van der, H. K., Goldschmeding, R., Logtenberg, T., and Clevers, H. Synergy between tumor suppressor APC and the $\beta$-catenm-Tcf4 target Tcfl. Science (Wash. DC), 285: 1923-1926, 1999.

17. Gradl, D., Kuhl, M., and Wedlich, D. The Wnt/Wg signal transducer $\beta$-catenin controls fibronectm expression. Mol. Cell. Biol., 19: $5576-5587,1999$.

18. Vallm, J., Thuret, R., Giacomello, E., Faraldo, M. M., Thiery, J. P., and Broders, F. Cloning and characterization of three Xenopus slug promoters reveal direct regulation by Lef/ $\beta$-catenm signaling. J. Biol. Chem., 276: 30350-30358, 2001.

19. Hlubek, F., Jung, A., Kotzor, N, Kirchner, T., and Brabletz, T. Expression of the invasion factor laminin $\gamma 2$ in colorectal carcinomas is regulated by $\beta$-catenin. Cancer Res., 61: 8089-8093, 2001

20. Steinert, P. M., and Roop, D. R. Molecular and cellular biology of intermediate filaments. Annu. Rev. Biochem, 57: 593-625, 1988.

21. Ramaekers, F. C, Haag, D., Kant, A., Moesker, O., Jap, P. H., and Vooijs, G. P. Coexpression of keratin- and vimentin-type intermediate filaments in human metastatic carcinoma cells. Proc. Natl. Acad. Sci. USA, 80: 2618-2622, 1983.

22. Guarino, M. Epithelial-to-mesenchymal change of differentiation. From embryoge-netic mechanism to pathological patterns. Histol. Histopathol., 10: 171-184, 1995.

23. Gilles, C, Polette, M., Piette, J., Delvigne, A. C, Thompson, E. W., Foidart, J. M., and Birembaut, P. Vimentin expression in cervical carcinomas: association with invasive and migratory potential. J. Pathol., 180: 175-180, 1996.

24. Gilles, C, Polette, M., Zahm, J. M., Tourmer, J. M., Volders, L., Foidart, J. M., and Birembaut, P. Vimentin contributes to human mammary epithelial cell migration. J. Cell Sci., 112 (Pt 24): 4615-4625, 1999.

25. Hendrix, M. J., Seftor, E. A., Seftor, R. E., and Trevor, K. T. Experimental co-expression of vimentin and keratin intermediate filaments in human breast cancer cells results in phenotypic interconversion and increased invasive behavior. Am. J. Pathol., 150: 483-495, 1997.

26. Eckes, B., Dogic, D., Colucci-Guyon, E., Wang, N, Maniotis, A., Ingber, D., Merckling, A., Langa, F., Aumailley, M., Delouvee, A., Koteliansky, V., Babinet, C, and Krieg, T. Impaired mechanical stability, migration and contractile capacity in vimentm-deficient fibroblasts. J. Cell Sci., 111 (Pt 13): 1897-1907, 1998

27. Eckes, B., Colucci-Guyon, E., Smola, H., Nodder, S., Babinet, C, Krieg, T., and Martin, P. Impaired wound healing in embryonic and adult mice lacking vimentin. J. Cell Sci., 113: 2455-2462, 2000.

28. Svitkina, T. M., Verkhovsky, A. B., and Borisy, G. G. Plectin sidearms mediate interaction of intermediate filaments with microtubules and other components of the cytoskeleton. J. Cell Biol., 135: 991-1007, 1996.

29. Maniotis, A. J., Chen, C. S., and Ingber, D. E. Demonstration of mechanical connections between integrins, cytoskeletal filaments, and nucleoplasm that stabilize nuclear structure. Proc. Natl. Acad. Sci. USA, 94: 849-854, 1997. 
30. Homan, S. M., Mercurio, A. M., and LaFlamme, S. E. Endothelial cells assemble two distinct $\alpha_{6} \beta_{4}$-containing vimentin-associated structures: roles for ligand binding and the $\beta_{4}$ cytoplasmic tail. J. Cell Sci., 111 (Pt 18): 2717-2728, 1998.

31. Goldman, R. D., Chou, Y. H., Prahlad, V., and Yoon, M. Intermediate filaments: dynamic processes regulating their assembly, motility, and interactions with other cytoskeletal systems. FASEB J., 13 (Suppl. 2): S261-S265, 1999.

32. Wu, A. L., Wang, J., Zheleznyak, A., and Brown, E. J. Ubiquitin-related proteins regulate interaction of vimentin intermediate filaments with the plasma membrane. Mol. Cell, 4: 619-625, 1999.

33. Gonzales, M., Weksler, B., Tsuruta, D., Goldman, R. D., Yoon, K. J., Hopkinson, S. B., Flitney, F. W., and Jones, J. C. Structure and function of a vimentin-associated matrix adhesion m endothelial cells. Mol. Biol. Cell, 12: 85-100, 2001.

34. Goldman, R. D., Khuon, S., Chou, Y. H., Opal, P., and Steinert, P. M. The function of intermediate filaments in cell shape and cytoskeletal integrity. J. Cell Biol., 134: 971-983, 1996.

35. Hendrix, M. J., Seftor, E. A., Chu, Y. W., Trevor, K. T., and Seftor, R. E. Role of intermediate filaments in migration, invasion and metastasis. Cancer Metastasis Rev., 15: 507-525, 1996.

36. Sommers, C. L., Byers, S. W., Thompson, E. W., Torri, J. A., and Gelmann, E. P. Differentiation state and invasiveness of human breast cancer cell lines. Breast Cancer Res. Treat, 31: 325-335, 1994

37. Polette, M., Gilles, C, de Bentzmann, S., Gruenert, D., Tournier, J. M., and Birembaut, P. Association of fibroblastoid features with the invasive phenotype in human bronchial cancer cell lines. Clin. Exp. Metastasis, 16: 105-112, 1998.

38. Martel, C, Harper, F., Cereghini, S., Noe, V., Mareel, M., and Cremisi, C. Inactiva-tion of retinoblastoma family proteins by SV40 T antigen results in creation of a hepatocyte growth factor/scatter factor autocrine loop associated with an epithelial-fibroblastoid conversion and invasiveness. Cell Growth Differ., 8: 165-178, 1997.

39. Rittling, S. R., and Baserga, R. Functional analysis and growth factor regulation of the human vimentin promoter. Mol. Cell. Biol., 7: $3908-3915,1987$

40. Korinek, V., Barker, N, Morin, P. J., van Wichen, D., de Weger, R., Kinzler, K. W, Vogelstein, B., and Clevers, H. Constitutive transcriptional activation by a $\beta$-catenin-Tcf complex m APC-/- colon carcinoma. Science (Wash. DC), 275: 1784-1787, 1997.

41. van de Wetering, M., W. M., Cavallo, R., Dooijes, D., van Beest, M., van Es, J., Loureiro, J., Ypma, A., Hursh, D., Jones, T., Bejsovec, A., Peifer, M., Mortin, M., and Clevers, H. Armadillo coactivates transcription driven by the product of the Dro-sophila segment polarity gene dTCF. Cell, 88: 789-799, 1997.

42. Orford, K., Crockett, C, Jensen, J. P., Weissman, A. M., and Byers, S. W. Serine phosphorylation-regulated ubiquitination and degradation of $\beta$-catenin. J. Biol. Chem., 272: 24735-24738, 1997.

43. Sommers, C. L., Walker-Jones, D., Heckford, S. E., Worland, P., Valverius, E., Clark, R., McCormick, F., Stampfer, M., Abularach, S., and Gelmann, E. P. Vimentin rather than keratin expression in some hormone-independent breast cancer cell lines and in oncogenetransformed mammary epithelial cells. Cancer Res., 49: 4258-4263, 1989.

44. Sommers, C. L., Thompson, E. W., Torri, J. A., Kemler, R., Gelmann, E. P., and Byers, S. W. Cell adhesion molecule uvomorulin expression in human breast cancer cell lines: relationship to morphology and invasive capacities. Cell Growth Differ., 2: 365-372, 1991.

45. Thompson, E. W., Paik, S., Brunner, N, Sommers, C. L., Zugmaier, G., Clarke, R., Shima, T. B., Torri, J., Donahue, S., and Lippman, M. E. Association of increased basement membrane invasiveness with absence of estrogen receptor and expression of vimentin in human breast cancer cell lines. J. Cell Physiol, 150: 534-544, 1992.

46. Muller, T., Choidas, A., Reichmann, E., and Ullrich, A. Phosphorylation and free pool of $\beta$-catenin are regulated by tyrosine kinases and tyrosine phosphatases during epithelial cell migration. J. Biol. Chem., 274: 10173-10183, 1999.

47. Eger, A., Stockinger, A., Schaffhauser, B., Beug, H., and Foisner, R. Epithelial mesenchymal transition by c-Fos estrogen receptor activation involves nuclear translocation of $\beta$-catenin and upregulation of $\beta$-catenin/lymphoid enhancer binding factor-1 transcriptional activity. J. Cell Biol., 148: 173-188, 2000.

48. Reichert, M., Muller, T., and Hunziker, W. The PDZ domains of zonula occludens-1 induce an epithelial to mesenchymal transition of Madin-Darby canine kidney I cells. Evidence for a role of $\beta$-catenin/Tcf/Lef signaling. J. Biol. Chem., 275: $9492-9500,2000$.

49. Kim, K., Lu, Z., and Hay, E. D. Direct Evidence for a Role of $\beta$-catenin/LEF-1 signaling pathway m induction of EMT. Cell Biol. Int., 26: $463-476,2002$.

50. Murakami, T., Toda, S., Fujimoto, M., Ohtsuki, M., Byers, H. R., Etoh, T., and Nakagawa, H. Constitutive activation of Wnt $/ \beta$-catenin signaling pathway in migration-active melanoma cells: role of LEF-1 in melanoma with increased metastatic potential. Biochem. Biophys Res. Commun., 288: 8-15, 2001. 
51. van de Wetering, M., Barker, N, Harkes, I. C, van der Heyden, M., Dijk, N. J, Hollestelle, A., Klijn, J. G., Clevers, H, and Schutte, M. Mutant E-cadherin breast cancer cells do not display constitutive Wnt signaling. Cancer Res., 61: 278-284, 2001.

52. Rittling, S. R., Coutinho, L., Amram, T., and Kolbe, M. AP-1/jun binding sites mediate serum inducibility of the human vimentin promoter. Nucleic Acids Res., 17: 1619-1633, 1989.

53. Van de Klundert, F. A., van Eldik, G. J., Pieper, F. R., Jansen, H. J., and Bloemendal, H. Identification of two silencers flanking an AP-1 enhancer in the vimentin promoter. Gene (Amst), 122: 337-343, 1992.

54. Lilienbaum, A., and Paulin, D. Activation of the human vimentin gene by the Tax human T-cell leukemia virus. I. Mechanisms of regulation by the NF- $\mathrm{KB}$ transcription factor. J. Biol. Chem., 268: 2180-2188, 1993.

55. Salvetti, A., Lilienbaum, A., Li, Z., Paulin, D., and Gazzolo, L. Identification of a negative element in the human vimentin promoter: modulation by the human T-cell leukemia virus type I Tax protein. Mol. Cell. Biol., 13: 89-97, 1993.

56. Van de Klundert, F. A., Jansen, H. J., and Bloemendal, H. Negative regulation of a special, double AP-1 consensus element in the vimentin promoter: interference by the retinoic acid receptor. J. Cell. Physiol., 164: 85-92, 1995.

57. Chen, J. H., Vercamer, C, Li, Z., Paulin, D., Vandenbunder, B., and Stehelin, D. PEA3 transactivates vimentin promoter in mammary epithelial and tumor cells. Oncogene, 13: 1667-1675, 1996.

58. Moura-Neto, V., Kryszke, M. H., Li, Z., Vicart, P., Lilienbaum, A., and Paulin, D. A 28-bp negative element with multiple factor-binding activity controls expression of the vimentin-encoding gene. Gene (Amst.), 168: 261-266, 1996.

59. Izmailova, E. S., and Zehner, Z. E. An antisilencer element is involved in the transcriptional regulation of the human vimentin gene. Gene (Amst.), 230: 111-120, 1999

60. Wieczorek, E., Lin, Z., Perkins, E. B., Law, D. J., Merchant, J. L., and Zehner, Z. E. The zinc finger repressor, ZBP-89, binds to the silencer element of the human vimentin gene and complexes with the transcriptional activator, Spl. J. Biol. Chem., 275: 12879-12888, 2000.

61. Pieper, F. R., Van de Klundert, F. A., Raats, J. M., Henderk, J. B., Schaart, G., Ramaekers, F. C, and Bloemendal, H. Regulation of vimentin expression in cultured epithelial cells. Eur. J. Biochem., 210: 509-519, 1992.

62. Sommers, C. L., Skerker, J. M., Chrysogelos, S. A., Bosseler, M., and Gelmann, E. P. Regulation of vimentin gene transcription in human breast cancer cell lines. Cell Growth Differ., 5: 839-846, 1994.

63. Cavallo, R. A., Cox, R. T., Moline, M. M., Roose, J., Polevoy, G. A., Clevers, H., Peifer, M., and Bejsovec, A. Drosophila Tcf and Groucho interact to repress Wingless signalling activity. Nature (Lond.), 395: 604-608, 1998.

64. Levanon, D., Goldstein, R. E., Bernstein, Y., Tang, H., Goldenberg, D., Stifani, S., Paroush, Z., and Groner, Y. Transcriptional repression by AML1 and LEF-1 is mediated by theTLE/Groucho corepressors. Proc. Natl. Acad. Sci. USA, 95: 11590-11595, 1998. 